\title{
ChemComm
}

\section{Transmission of photo-catalytic function in a self-replicating chemical system: in situ amphiphile production over two protocell generations $\dagger$}

Cite this: Chem. Commun., 2014, 50,8989

Received 28th February 2014 Accepted 25th June 2014

DOI: $10.1039 / c 4 c c 01543 f$

\author{
A. N. Albertsen, ${ }^{a}$ S. E. Maurer, ${ }^{b}$ K. A. Nielsen ${ }^{a}$ and P.-A. Monnard*a
}

www.rsc.org/chemcomm

\begin{abstract}
Glass microsphere supported protocells were built to investigate the transmission of catalytic function during replication. The chemical system's replication was driven through in situ amphiphile production that resulted in the formation of free bilayers, the system's second "generation". It was demonstrated that both generations, once separated, still exhibited the ability to convert amphiphile precursors. This result shows that transmission of function in chemical systems is possible during self-replication.
\end{abstract}

Morphological alterations of chemical systems induced by the addition of building blocks are essential in various applications of systems chemistry ranging from protocells (i.e., simplified models for cellular ${ }^{2,3}$ or precellular systems ${ }^{4}$ ) to "smart" materials that perform a function upon environmental triggering. ${ }^{5}$ Several aspects of protocell replication remain yet unexplored, among others the transmission of properties between the original chemical system and its "off-spring" and the effect of the growth and division rates on this transmission. In the former challenge, the difficulty often resides in the observer's ability to distinguish between or/and separate the different "generations." In the latter, the choice of building block sources (i.e., external addition ${ }^{6}$ or in situ production of material $\left.{ }^{2,7}\right)$ is paramount as it will define the growth/division rates and by extension the time available for the equilibration of system composition, i.e., the distribution of molecular components into the newly created system boundaries or volumes.

One protocell system developed by DeClue et $a l^{1}{ }^{1}$ offers the opportunity to study the replication process upon in situ production of building blocks (see ESI†). This protocell model incorporated

\footnotetext{
${ }^{a}$ Institute for Physics, Chemistry and Pharmacy, University of Southern Denmark, Campusvej, 55, 5230 Odense M, Denmark.E-mail: monnard@sdu.dk

${ }^{b}$ Department of Chemistry and Biochemistry, Central Connecticut State University, 1615 Stanley St., New Britain, CT 06050, USA

$\dagger$ Electronic supplementary information (ESI) available: It contains a description of experiments, additional data supporting the construction of the microspheres supported decanoic acid bilayers, the interactions between the both ruthenium complex and the precursor lipid with the supported bilayers. There are additional figures and micrographs of the growth and division process of the system. See DOI: $10.1039 / \mathrm{c} 4 \mathrm{cc} 01543 \mathrm{f}$
}

decanoic acid bilayers self-assembled into vesicles, as protocell compartments, and a photochemical, catalytic, amphiphile production apparatus, which is composed of a ruthenium complex (photosensitizer and redox catalyst), a modified nucleobase, 8-oxoguanine (acting as electron relay) and dihydrogen phenylglycine (the hydrogen donor). This system's ability to photochemically convert $N$-methylpicolinium decanoate (the precursor lipid) into decanoic acid has been investigated in bulk ${ }^{1}$ and in association with decanoic acid vesicles. ${ }^{8}$

This protocell system is capable of generating amphiphiles rapidly enough to allow the growth process to be monitored. A complete description of the process is given in the ESI. $\dagger$ In this paper, the photochemical reaction is used to produce enough fatty acid to enable the growth and division of decanoic acid membranes. This reaction is entirely contained in the bilayers themselves by the hydrophobicity of the reactant, catalyst and product. Thus the compartment is the bilayer and not the aqueous interior of the vesicle (Fig. 1).

The division process has yet to be studied with this photochemical amphiphile catalytic system. The challenges with decanoic acid structures are rooted in their intrinsic properties: for example, even extruded vesicles spontaneously undergo fusion and fission at room temperature. That is, a certain homogenization of the lipid bilayer composition and high polydispersity cannot be prevented during long incubation periods. ${ }^{9}$

Despite these drawbacks, the use of decanoic acid structures is central for the design of protocells as models for the emergence of precellular systems in origins-of-life scenarios. This amphiphile is indeed considered prebiotically plausible after its detection in the organic content of meteorite $\mathrm{s}^{10-12}$ and its synthesis in FischerTropsch type reactions, which emulate the plausible prebiotic synthetic pathways. ${ }^{13,14}$

To circumvent the challenges presented by decanoic acid structures, a novel self-assembled aggregate was developed (Fig. 1), which is based on anchoring of the bilayer membranes to glass microspheres. This approach presents several advantages: (i) the polydispersity of these hybrid structures is determined by the low polydispersity of the microspheres; (ii) the anchoring of the bilayer 


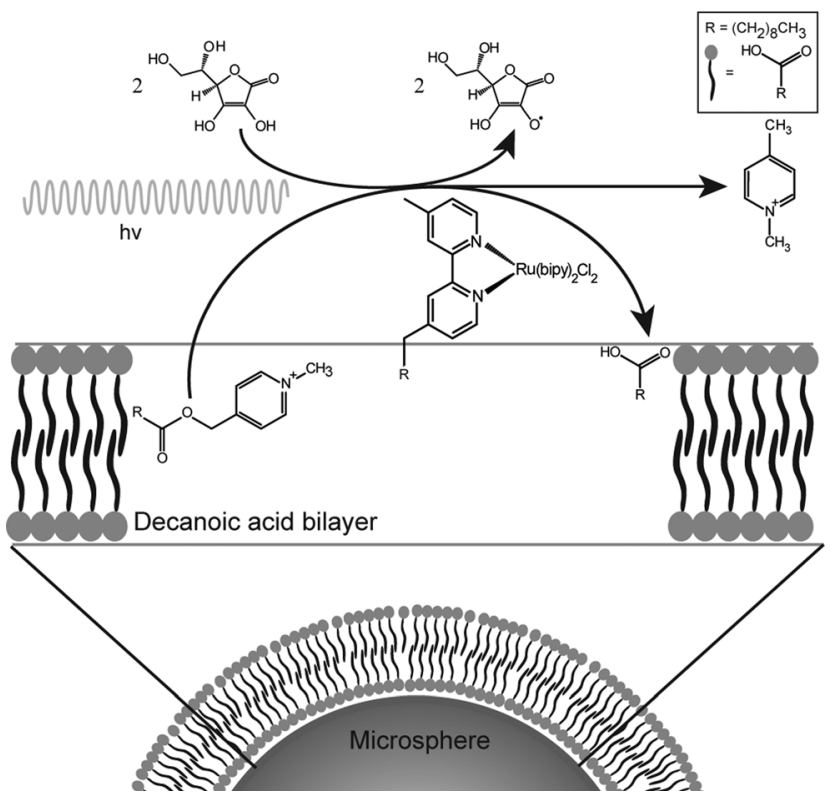

Fig. 1 Schematic representation of the chemical system and the associated amphiphile production. The amphiphile symbols represent decanoic acid molecules.

increases the stability of the membrane, (iii) the density of the microspheres permits the exchange of the hosting solution by sedimentation methods such as centrifugation; and (iv) the separation of division products, present as unsupported vesicles and tubes, and the microsphere-supported bilayers that produced them is likewise achievable. Thus, the separation and characterization of two protocell generations and their individual properties are possible.

The anchoring of phospholipid bilayers to $\mathrm{SiO}_{2}$ microspheres has been described by Gopalakrishnan et al..$^{15}$ and was modified to accommodate decanoic acid bilayers. The bilayer anchoring was achieved by coating the microspheres with avidin and incubating them with a lipid mixture consisting of decanoic acid and biotinylated lipid, with a mole ratio of DA: biotinylated lipid of 3954:1. Using Nile red, a hydrophobic fluorescent dye, it was established that all microspheres were coated with anchored bilayers by microscopic analysis. Interestingly, the critical decanoic acid concentration for complete microsphere coating was $0.421 \mathrm{mM}$ $\pm 0.059 \mathrm{mM}$, see $\mathrm{ESI} \dagger$ (Fig. SG), whereas decanoic acid has a critical vesicle concentration of $10.7 \pm 1.6 \mathrm{mM}$ in bulk. ${ }^{9}$ Nevertheless, the supported bilayers remained stable over time and were resistant to exchanges of the solution hosting the microspheres, in clear contrast to a coating in the absence of avidin/biotinylated lipid. This strong interaction between the microspheres and the bilayers was a prerequisite for the observation of the division process, see ESI $\dagger$ (Fig. SF and SG).

Assuming the same headgroup area $\left(22 \AA^{2}\right)$ as for stearic acid, ${ }^{16}$ the coating procedure resulted in a multilamellar bilayer configuration on the microsphere surface with approximately $4.68 \times 10^{-14}$ $\pm 6.56 \times 10^{-15}$ mole decanoic acid for each microsphere, which corresponds to an average of 39 bilayers per microsphere. Multilamellarity is a known property of self-assembled aggregates, and has been reported in supported bilayers ${ }^{17}$ and vesicles.
In order to catalyse the conversion of precursor lipid, the ruthenium complex had to be strongly associated with the anchored bilayer. The amphiphilic catalyst [Ru(II)(bpy) 2 (4-decyl,4'-methyl-bpy)] chloride (RuC 10:0) was added in the aqueous medium and spontaneously inserted into the bilayers. On average, the ruthenium complex was associated with each microsphere at a molar ratio of catalyst to lipid of $47: 1$. This ratio corresponded to the maximum amount of which a bead could accommodate, see ESI $\dagger$ (Fig. SH).

The insertion of the ruthenium complex into the supported bilayers did not inhibit the cleavage of a precursor lipid into decanoic acid by the photocatalyst, as it proceeded as previously described by ref. 1 and 8. Due to the catalyst's high sensitivity to oxygen in the aqueous solution, a modified version of the photochemical system was implemented, which relied on ascorbic acid as both the electron and hydrogen donor (Fig. 1). Compared to the original system, the catalyst system with ascorbic acid showed significantly faster reaction rates, see ESI $\dagger$ (Fig. SM and SQ). This modification, when compared with the original system, can be explained by the different catalyst concentration in each set-up: $1 \mu$ mole catalyst for $600 \mu$ mole lipid precursor in this study compared to $1 \mu$ mole for 5 to $50 \mu$ mole in the previous studies. ${ }^{1,8}$ The ascorbic acid set-up was applied for all photochemical conversions of precursor lipid used to study growth and division.

During the reported experiments, a concentrated ethanol solution of precursor lipid, $\mathrm{N}$-methylpicolinium decanoate, was slowly injected into a vial containing ascorbic acid and the microsphere based protocellular system. Such a procedure led to almost complete insertion of precursor lipid into the supported bilayers, ensuring the photochemistry would occur in the bilayers, for a detailed description of the interaction see $\mathrm{ESI} \dagger$ (Fig. SL). Illuminating the reaction mixture for 168 hours resulted in the almost quantitative photochemical conversion of $12 \mathrm{mM}$ precursor lipid by the ruthenium complexes associated with the bilayers anchored to the microspheres, Fig. 2. HPLC analysis of the reaction showed that the majority of the precursor lipid was converted via the photochemical pathway while the non-catalytic hydrolysis of the lipid precursor remained low, see also ESI $\dagger$ (Fig. SP). Micrographs obtained at the end of the reaction show both vesicles as well as microspheres with attached bilayers structures, such as vesicles and tubular structures in the reaction mixture, Fig. 3.

To separate the microspheres from newly formed vesicles, the reaction mixture was centrifuged for 10 minutes at $6000 \mathrm{rpm}$. Centrifugation of this solution permitted a complete separation of vesicles from the supported bilayers, Fig. 4.

Having demonstrated that two protocell generations were distinguishable and could be effectively separated, the individual reactivity of each system was assessed. Fresh ascorbic acid and precursor lipid were added to each system in order to replenish the supply of substrates and co-factors. Each of the samples were then separately illuminated and analysed by HPLC, Fig. 2 . The sample analysis showed that the second generation of either microsphere systems or vesicles was capable of precursor lipid conversion. The primary reaction pathway remained photocatalysis, during the second illumination, as indicated by the low concentrations of hydrolysis waste product in the HPLC analysis. Micrographs of the vesicle suspension showed membranous structures, as well as oil 


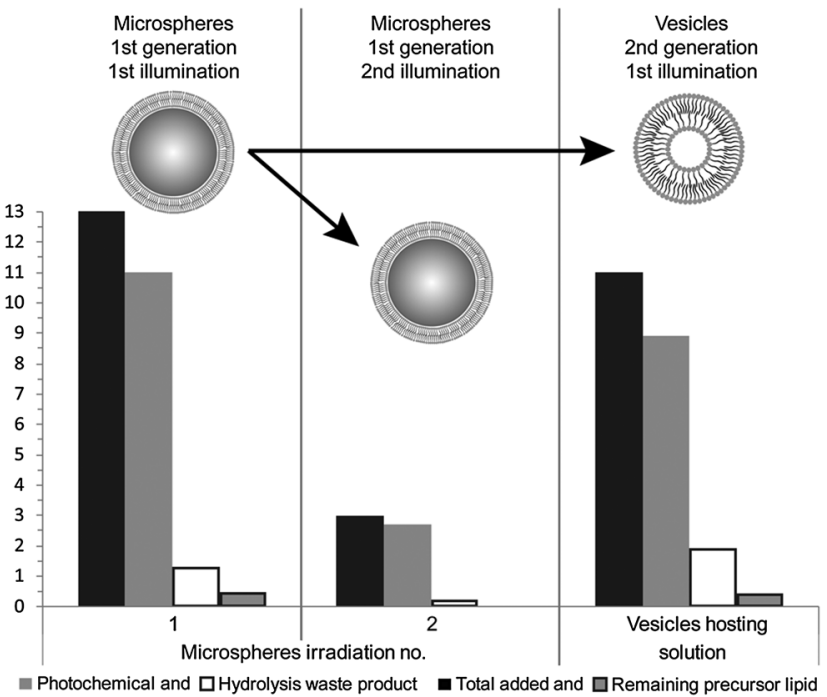

Fig. 2 Photochemical conversion of precursor lipid. The conversion yields are displayed for the original microsphere system after its first illumination, as well as for the microspheres after a second illumination and vesicles collected in the supernatant after centrifugation after their first illumination (two last systems representing the second generation of protocell). A bulk addition of precursor lipid was employed to re-supply the vesicle and microsphere systems during their first and second illumination.

droplets of the precursor lipid whose presence in significant amounts was confirmed by the HPLC analysis, Fig. 2 and 4 .

The production of vesicles likely occurred through budding. Indeed, protruding bilayer tubes on the surface of the bilayercoated microspheres can be clearly observed in the micrograph A of Fig. 3.

Some vesicles were observed in solution before centrifugation, however the centrifugation process ensured a complete disappearance of the tubes attached on the microspheres, expediting the division process, Fig. 4 . The forces experienced during centrifugation by the system likely induced vesicle splitting of the remaining tubes in a manner similar to the mechanism reported by Zhu and Szostak. ${ }^{18}$ These authors demonstrated that small tubes could be split into distinct vesicles upon gentle shaking.

The transmission of the photochemical function could have happened via two simultaneous but distinct pathways: (i) the initially inserted ruthenium catalysts would be separated from the microsphere supported protocell along with protruding bilayers, Fig. 3A, or (ii) the ruthenium could have dissociated from these bilayers and then associated with other structures. Its passive exchange was observed between microspheres and vesicles at high concentrations of vesicles, but not between microspheres and lipid oil droplets, see $\mathrm{ESI} \dagger$ (Fig. SN).

Interestingly, a conversion rate increase only occurred after the formation of vesicles in the sample, indicating that the reaction surface, i.e., the number of catalyst molecules capable of interaction with the ascorbic acid in the medium, had increased, see ESI $\dagger$ for more detailed information (Fig. SP). This observation supports the hypothesis that the transmission of the photocatalyst during the budding process, and not as diffusion through the hosting solution. Such a trend would be expected if the budding of vesicles uncovered
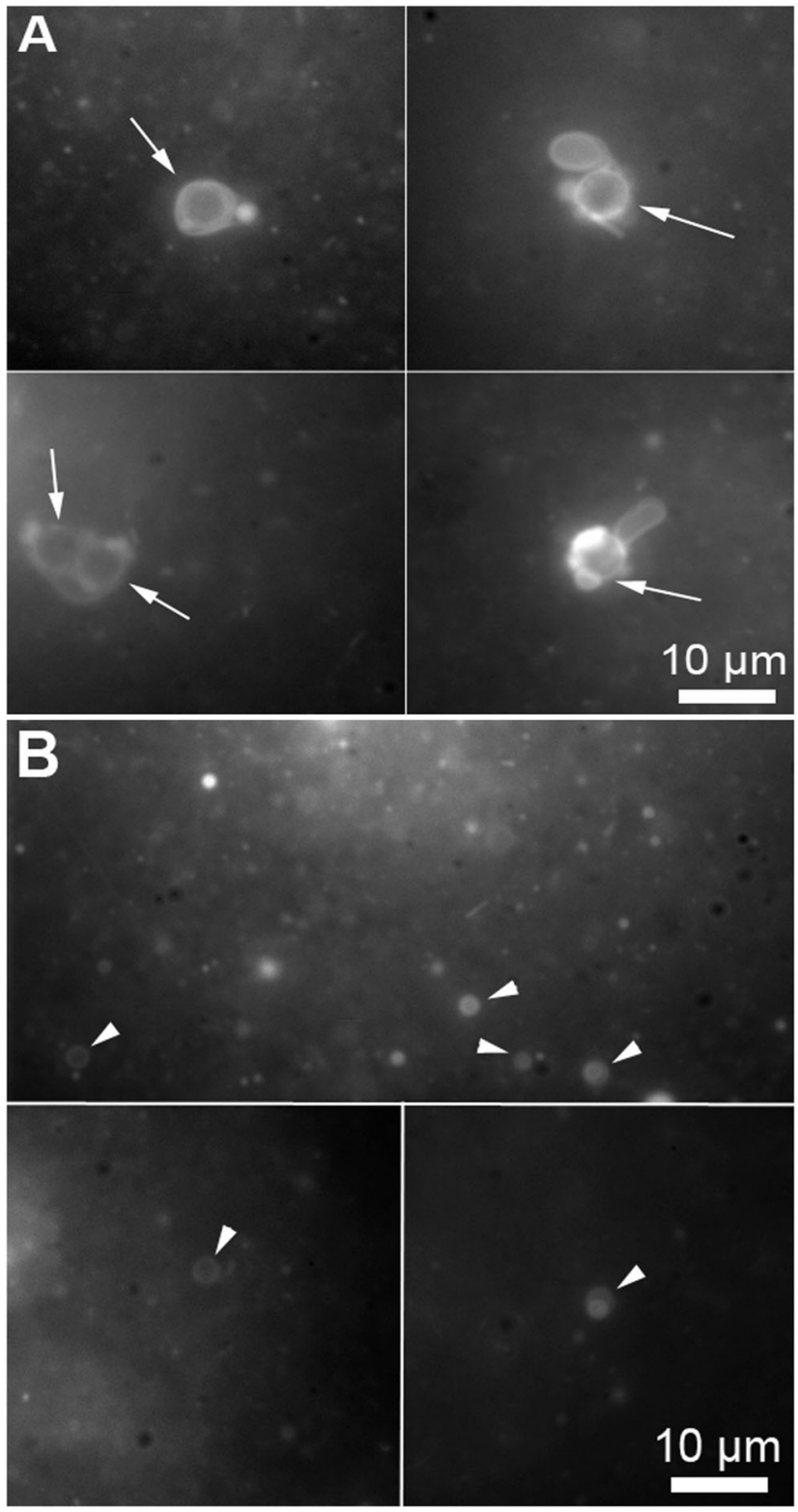

Fig. 3 Micrographs of a microsphere solution after the photochemical conversion of precursor lipid, the conversion is quantified in Fig. 2. (A) Bilayer structures associated with microspheres (the arrows points to the microspheres). The microspheres are identifiable by the circular shape and their specific size $(5 \mu \mathrm{m})$. (B) Vesicles produced during the conversion of the fatty acid precursors on the microspheres (the arrow heads point to free floating vesicles). The micrographs were taken from samples containing both microspheres and free floating vesicles stained with $0.1 \mathrm{mM}$ Nile red.

new catalysts associated with bilayers previously hidden away from the medium, clearly a possibility, considering the average number of bilayers per microsphere.

It is likely that the ruthenium catalyst in the vesicle bilayers was predominantly "transmitted" as components of the tube bilayers that split into the vesicles, the second generation of protocells. Although these experiments are focusing on a rather simple chemical system (one single function), they demonstrate that a second system generation can retain essential properties of the first generation, i.e., the general system identity, after division. 


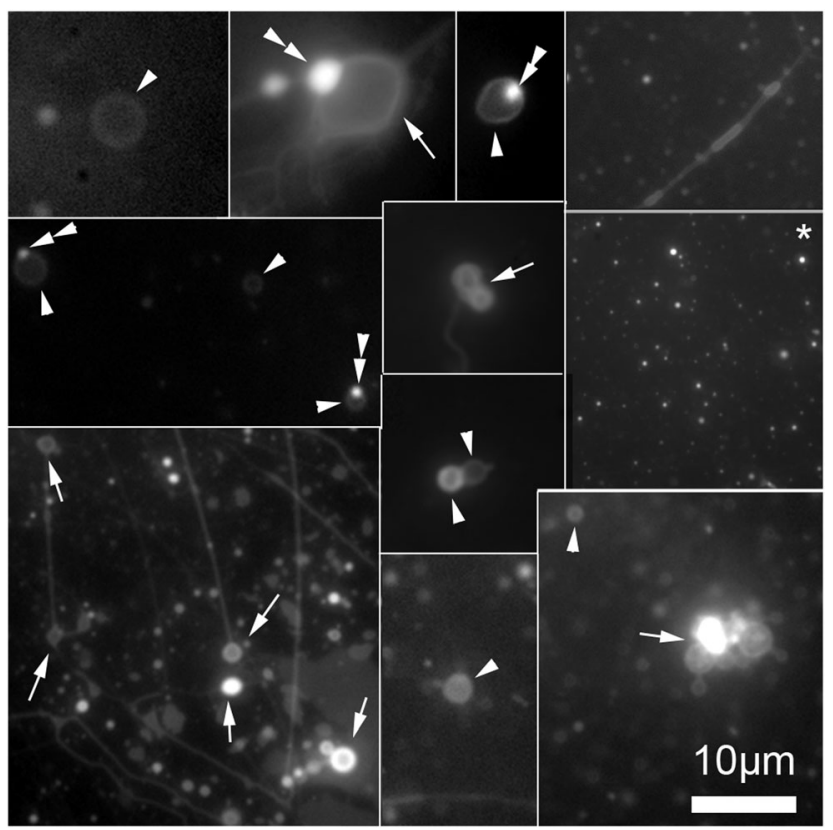

Fig. 4 Collage of cropped micrographs depicting the various structures found in the hosting solution after removal of the microspheres by centrifugation. The arrow heads points to freely floating vesicles. Some of these vesicles have oil inclusions (double arrow heads) in their membranes resulting in brighter spots in these membranes. The arrows point to structures stuck the microscope slide. Among the structures observed are also bilayer tubes (top right and bottom left micrographs). The micrograph marked with an asterisk (*) present precursor lipid oil droplet after the separation from the microspheres. The presence of the lipid precursor as well as the photochemical waste product was confirmed by the HPLC analysis, Fig. 2. The micrographs were stained with $0.1 \mathrm{mM}$ Nile red.

In this communication, we demonstrated that it was possible to anchor decanoic acid bilayers onto glass microspheres using the interaction between avidin and a minute amount of biotinylated lipid solubilised into the decanoic acid bilayers. Inserting a ruthenium based catalytic apparatus into these bilayers permitted the photochemical conversion of the precursor lipid into the membrane amphiphiles. Conversion yields were sufficient to induce vesicle formation. These vesicles could then be separated from the microsphere supported bilayers by centrifugation. After replenishing their substrate supply, vesicles and anchored bilayer systems continued to efficiently perform the photochemical conversion. Thus, the catalytic capability of the original protocells (microspheres supported bilayers) was transmitted to novel protocells (vesicles), products of the replication of the original systems.

The authors would like to acknowledge M. S. Declue and M. Dörr. They are grateful for many fruitful discussions from colleagues at the University of Southern Denmark (SDU) and Los Alamos National Laboratory. This work was supported by the FLinT Center at SDU, SDU, and the EC sponsored project (Matchit \#249032).

\section{Notes and references}

1 M. S. DeClue, P. A. Monnard, J. A. Bailey, S. E. Maurer, G. E. Collis, H. J. Ziock, S. Rasmussen and J. M. Boncella, J. Am. Chem. Soc., 2009, 131, 931-933.

2 Y. Kuruma, P. Stano, T. Ueda and P. L. Luisi, Biochim. Biophys. Acta, Biomembr., 2009, 1788, 567-574.

3 H. Kita, T. Matsuura, T. Sunami, K. Hosoda, N. Ichihashi, K. Tsukada, I. Urabe and T. Yomo, ChemBioChem, 2008, 9, 2403-2410.

4 K. Adamala and J. W. Szostak, Nat. Chem., 2013, 5, 495-501.

5 M. A. C. Stuart, W. T. S. Huck, J. Genzer, M. Muller, C. Ober, M. Stamm, G. B. Sukhorukov, I. Szleifer, V. V. Tsukruk, M. Urban, F. Winnik, S. Zauscher, I. Luzinov and S. Minko, Nat. Mater., 2010, 9, 101-113.

6 P. Stano, E. Wehrli and P. L. Luisi, J. Phys.: Condens. Matter, 2006, 18, S2231-S2238.

7 T. F. Zhu, K. Adamala, N. Zhang and J. W. Szostak, Proc. Natl. Acad. Sci. U. S. A., 2012, 109, 9828-9832.

8 S. E. Maurer, M. S. DeClue, A. N. Albertsen, M. Dörr, D. S. Kuiper, H. Ziock, S. Rasmussen, J. M. Boncella and P.-A. Monnard, ChemPhysChem, 2011, 12, 828-835.

9 S. E. Maurer, D. W. Deamer, J. M. Boncella and P. A. Monnard, Astrobiology, 2009, 9, 979-987.

10 A. A. Monroe and S. Pizzarello, Geochim. Cosmochim. Acta, 2011, 75, 7585-7595.

11 S. Pizzarello and W. Holmes, Geochim. Cosmochim. Acta, 2009, 73, 2150-2162.

12 M. N. Mautner, R. L. Leonard and D. W. Deamer, Planet. Space Sci., 1995, 43, 139-147.

13 T. M. McCollom, G. Ritter and B. R. T. Simoneit, Orig. Life Evol. Biosph., 1999, 29, 153-166.

14 A. I. Rushdi and B. R. T. Simoneit, Orig. Life Evol. Biosph., 2001, 31, 103-118.

15 G. Gopalakrishnan, I. Rouiller, D. R. Colman and R. B. Lennox, Langmuir, 2009, 25, 5455-5458.

16 N. Rontu and V. Vaida, J. Phys. Chem. C, 2007, 111, 9975-9980.

17 T. A. Oleson and N. Sahai, Langmuir, 2008, 24, 4865-4873.

18 T. F. Zhu and J. W. Szostak, J. Am. Chem. Soc., 2009, 131, 5705-5713. 